\title{
Using Phase Relations in Microstrip Directional Couplers to Achieve High Directivity
}

\author{
Vahdettin Tas and Abdullah Atalar, Fellow, IEEE
}

\begin{abstract}
We analyze the microstrip directional couplers with a special focus on the phase difference between the coupled and isolated ports. The analysis uses the even-odd mode decomposition technique and network theory. The results show that the phase difference between the signals at the coupled port and the isolated port is close to $\pi$ in a very wide band. This property can be used to achieve a broadband directivity by inducing a voltage cancellation at the isolated port of the couplers. Two different cancellation methods are investigated. Analytical formulas for the required component values of both methods are given. Higher directivity can be obtained at the expense of reduced bandwidth.
\end{abstract}

Index Terms-Broadband microstrip coupler, coupler phase relations, coupling isolation phase difference, high directivity.

\section{INTRODUCTION}

$\mathbf{P}$ LANAR characteristic of the microstrip line technology offers substantial advantages with a low cost of fabrication and integrability with other components. The propagation mode in a microstrip line is quasi-TEM, because the medium is inhomogeneous. The quasi-TEM mode propagation is especially problematic when coupled line directional couplers are under consideration. The inhomogeneity of the medium causes the even and odd modes to propagate with different velocities that degrade directivity [1].

There are several methods to compensate the even mode odd mode velocity differences in microstrip line directional couplers. Podell [2] introduced the wiggly line coupler. In that design, the odd mode travels a longer path; hence it is slowed down relative to the even mode. Dielectric overlays [3], [4] achieves a velocity equalization as the overlay brings the quasi-TEM mode propagation closer to a pure TEM mode. Lumped element compensation is explored in several works [5]-[7]. Moderate bandwidth and directivity levels were achieved using these methods. A recent work [8] demonstrated a wideband compensation using interdigital capacitors. In another recent work [9] both high directivity and tight coupling were achieved by cascading couplers in a narrow bandwidth. High values of directivity improvement were obtained using voltage cancellation methods. In [10], [11] termination impedances were adjusted to cancel the transmission to the isolated port. Feedforward compensation was applied in [12], [13]. Bypass circuits were utilized in [14]. The techniques based on voltage cancellation [10]-[14]

Manuscript received May 04, 2013; revised October 22, 2013; accepted October 24, 2013. Date of publication November 13, 2013; date of current version December 02, 2013.

The authors are with the Department of Electrical and Electronics Engineering, Bilkent University, Ankara 06800, Turkey (e-mail: vtas@ee.bilkent. edu.tr; aatalar@bilkent.edu.tr).

Color versions of one or more of the figures in this paper are available online at http://ieeexplore.ieee.org.

Digital Object Identifier 10.1109/TMTT.2013.2288601

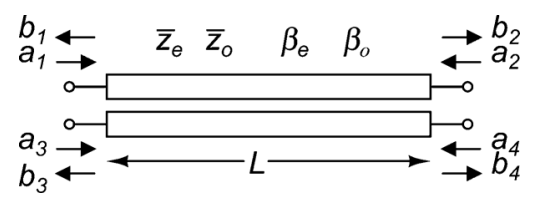

Fig. 1. Microstrip coupler of length $L$ with normalized even $\left(\bar{z}_{e}\right)$ and odd $\left(\bar{z}_{o}\right)$ mode characteristic impedances. $\beta_{e}$ and $\beta_{o}$ are even and odd mode propagation constants. $a_{i}$ and $b_{i}$ represent the incident and reflected wave amplitudes at port $i$.

achieved high directivity values, however the frequency range of the directivity improvement was narrowband relative to the quarter wavelength frequency.

The phase relations between the coupled port and the isolated port have not been explored in the literature. To the authors' knowledge, there is only one reference [13] to a $\pi$ phase difference for a quarter wavelength coupler design, but no analysis is presented about the frequency or dimensional dependence.

Using even-odd mode analysis, we show that the phase difference between the coupling factor and the isolation factor is close to $\pi$ from DC up to the half wavelength frequency. Network analysis also yields that complex isolation factor and the coupling factor of a microstrip coupled line coupler lie along the same vector. This fact presents a high potential to achieve broadband directivity improvement through a voltage cancellation at the isolated port.

This paper aims to obtain wide band high directivity by improving the voltage cancellation methods in the light of the new S-parameter phase relations. Two coupler architectures are investigated for improvement. Using the proposed method, the directivity bandwidth of couplers is significantly increased.

\section{EVEn-ODD Mode ANALYSIS}

Consider a lossless symmetrical microstrip coupler shown in Fig. 1. We write the S-parameter matrix of the coupler as

$$
\left[\begin{array}{l}
b_{1} \\
b_{2} \\
b_{3} \\
b_{4}
\end{array}\right]=\left[\begin{array}{llll}
\Gamma & T & C & I \\
T & \Gamma & I & C \\
C & I & \Gamma & T \\
I & C & T & \Gamma
\end{array}\right]\left[\begin{array}{l}
a_{1} \\
a_{2} \\
a_{3} \\
a_{4}
\end{array}\right]
$$

where $\Gamma, T, C, I$ are the reflection, transmission, coupling and isolation coefficients, respectively, all being complex numbers. This coupler can be analyzed using the even-odd mode analysis technique [1], [15], [16]. We can write

$$
\begin{aligned}
\Gamma & =\frac{1}{4}\left(\Gamma_{e e}+\Gamma_{e o}+\Gamma_{o e}+\Gamma_{o o}\right) \\
C & =\frac{1}{4}\left(\Gamma_{e e}+\Gamma_{e o}-\Gamma_{o e}-\Gamma_{o o}\right) \\
I & =\frac{1}{4}\left(\Gamma_{e e}-\Gamma_{e o}-\Gamma_{o e}+\Gamma_{o o}\right)
\end{aligned}
$$


where

$$
\begin{gathered}
\Gamma_{e e}=\frac{-j \bar{z}_{e} \cot \left(\frac{\beta_{e} L}{2}\right)-1}{-j \bar{z}_{e} \cot \left(\frac{\beta_{e} L}{2}\right)+1} \\
\Gamma_{e o}=\frac{j \bar{z}_{e} \tan \left(\frac{\beta_{e} L}{2}\right)-1}{j \bar{z}_{e} \tan \left(\frac{\beta_{e} L}{2}\right)+1} \\
\Gamma_{o e}=\frac{-j \bar{z}_{o} \cot \left(\frac{\beta_{o} L}{2}\right)-1}{-j \bar{z}_{o} \cot \left(\frac{\beta_{o} L}{2}\right)+1} \\
\Gamma_{o o}=\frac{j \bar{z}_{o} \tan \left(\frac{\beta_{o} L}{2}\right)-1}{j \bar{z}_{o} \tan \left(\frac{\beta_{o} L}{2}\right)+1}
\end{gathered}
$$

$\bar{z}_{e}$ and $\bar{z}_{o}$ are the even and odd mode characteristic impedances of the coupled lines normalized with respect to $Z_{0}$ (the reference impedance for the S-parameters). $\beta_{e}$ and $\beta_{o}$ are the even and odd mode propagation constants of coupler of length $L . \beta_{e} / \beta_{o}$ is assumed to be constant throughout the frequency range. ${ }^{1}$ Moreover, we consider couplers with low coupling levels $(|C|<$ $-10 \mathrm{~dB}$ ), so that $1<\bar{z}_{e}<1.4$ and $0.7<\bar{z}_{o}<1$.

Let $f_{1}$ be the frequency where $\beta_{e} L=\pi / 4$ is satisfied, i.e., $f_{1}$ is the frequency where the coupler line length is equal to one eighth of the even mode wavelength. For a better comprehension of the expressions (2), (3) and (4), we simplify them at specific frequencies: $f=0$ and $f=2 f_{1}$.

\section{A. For $f=0$}

Using Taylor expansion of $(2)$ around $f=0$ (i.e., for $f \ll f_{1}$ ) we find

$\Gamma \approx j \frac{\pi}{16}\left(\frac{f}{f_{1}}\right)\left(\frac{\beta_{o}}{\beta_{e}} \bar{z}_{o}+\bar{z}_{e}-\frac{\beta_{o}}{\beta_{e}} \frac{1}{\bar{z}_{o}}-\frac{1}{\bar{z}_{e}}\right)$ for $f \ll f_{1}$

Equating (7) to zero gives the optimal relationship between the parameters.

$$
\frac{\bar{z}_{o} \beta_{o}+\bar{z}_{e} \beta_{e}}{\bar{z}_{e} \beta_{o}+\bar{z}_{o} \beta_{e}} \bar{z}_{o} \bar{z}_{e}=1
$$

If $\beta_{e}=\beta_{o}$, we must choose $\bar{z}_{e} \bar{z}_{o}=1$ to satisfy the condition. On the other hand, if $\beta_{e}>\beta_{o}$ which is the case for microstrip coupled lines, we find that $\bar{z}_{e} \bar{z}_{o}<1 . \bar{z}_{e}$ can be explicitly written in terms of $\bar{z}_{o}$ and $b=\beta_{e} / \beta_{o}$ as

$$
\bar{z}_{e}=\frac{1}{2 b}\left[\left(\frac{1}{\bar{z}_{o}^{2}}+4 b^{2}-2+\bar{z}_{o}^{2}\right)^{1 / 2}+\frac{1}{\bar{z}_{o}}-\bar{z}_{o}\right] .
$$

Alternatively, we can express $b$ in terms of $\bar{z}_{e}$ and $\bar{z}_{o}$ under optimal condition of (8) as

$$
b=\frac{\left(1-\bar{z}_{o}^{2}\right) \bar{z}_{e}}{\left(\bar{z}_{e}^{2}-1\right) \bar{z}_{o}}
$$

${ }^{1}$ Typically, this ratio does not vary more than $5 \%$ from the mean value [17]
We can write the first two terms of the Taylor series around $f=0$ for the complex coupling and isolation coefficients as

$$
\begin{aligned}
C \approx & j \frac{\pi}{16}\left(\frac{f}{f_{1}}\right)\left(\bar{z}_{e}-\frac{1}{\bar{z}_{e}}-\frac{\bar{z}_{o}}{b}+\frac{1}{b \bar{z}_{o}}\right) \\
& +\frac{\pi^{2}}{128}\left(\frac{f}{f_{1}}\right)^{2}\left(\bar{z}_{e}^{2}-\frac{1}{\bar{z}_{e}^{2}}+\frac{1}{b^{2} \bar{z}_{o}^{2}}-\frac{\bar{z}_{o}^{2}}{b^{2}}\right) \\
I \approx & -j \frac{\pi}{16}\left(\frac{f}{f_{1}}\right)\left(\bar{z}_{e}+\frac{1}{\bar{z}_{e}}-\frac{\bar{z}_{o}}{b}-\frac{1}{b \bar{z}_{o}}\right) \\
& -\frac{\pi^{2}}{128}\left(\frac{f}{f_{1}}\right)^{2}\left(\bar{z}_{e}^{2}+\frac{1}{\bar{z}_{e}^{2}}-\frac{1}{b^{2} \bar{z}_{o}^{2}}-\frac{\bar{z}_{o}^{2}}{b^{2}}\right) .
\end{aligned}
$$

Directivity for $f \ll f_{1}, D_{0}=|C / I|$, can be written as

$$
D_{0} \approx \frac{b \bar{z}_{e}-\frac{b}{\bar{z}_{e}}-\bar{z}_{o}+\frac{1}{\bar{z}_{o}}}{b \bar{z}_{e}+\frac{b}{\bar{z}_{e}}-\bar{z}_{o}-\frac{1}{\bar{z}_{o}}} .
$$

The phase difference between the coupling and isolation coefficients is given by

$$
(\angle C-\angle I) \approx \pi+\frac{\pi}{4} A B \frac{A^{\prime}-B^{\prime}}{A^{2}-B^{2}}\left(\frac{f}{f_{1}}\right) \text { for } f \ll f_{1}
$$

with

$$
\begin{aligned}
& A=b \bar{z}_{e}-\bar{z}_{o}, \quad A^{\prime}=\bar{z}_{e}+\frac{\bar{z}_{o}}{b} \\
& B=\frac{1}{\bar{z}_{o}}-\frac{b}{\bar{z}_{e}}, \quad B^{\prime}=\frac{1}{b \bar{z}_{o}}+\frac{1}{\bar{z}_{e}} .
\end{aligned}
$$

In (14), the term in front of $\left(f / f_{1}\right)$ vanishes for $B=0$ or $A^{\prime}=B^{\prime}$. These imply

$$
\frac{\bar{z}_{e}}{\bar{z}_{o}}=b \quad \text { or } \quad \frac{\bar{z}_{o}+b \bar{z}_{e}}{\bar{z}_{e}+b \bar{z}_{o}} \bar{z}_{o} \bar{z}_{e}=1
$$

The second condition in (17) is the same as (8) so we have $|\Gamma| \approx$ 0 and $(\angle C-\angle I) \approx \pi$. Even if (17) is not exactly satisfied, (14) still predicts a phase very close to $\pi$ since $\left(A^{\prime}-B^{\prime}\right) \ll 1$.

B. For $f=2 f_{1}$

At this frequency we have $\beta_{e} L=\pi / 2$. Hence, $\tan \left(\beta_{o} L / 2\right)=\tan \left(\pi / 4-\left(\beta_{e}-\beta_{o}\right) L / 2\right) \approx 1-\left(\beta_{e}-\beta_{o}\right) L$ with comparable $\beta_{e}$ and $\beta_{o}$. In this case (5) and (6) reduce to

$$
\begin{aligned}
\Gamma_{e e} & =\frac{-j \bar{z}_{e}-1}{-j \bar{z}_{e}+1}, \Gamma_{e o}=\frac{j \bar{z}_{e}-1}{j \bar{z}_{e}+1} \\
\Gamma_{o e} & \approx \frac{-j \bar{z}_{o}(1+\xi)-1}{-j \bar{z}_{o}(1+\xi)+1}, \Gamma_{o o} \approx \frac{j \bar{z}_{o}(1-\xi)-1}{j \bar{z}_{o}(1-\xi)+1}
\end{aligned}
$$

where $\xi=\left(\beta_{e}-\beta_{o}\right) L=(\pi / 2)(b-1) / b$. Combining (18) and (19) with (3) and (4), the ratio of the coupling coefficient to the isolation coefficient can be written as

$$
\frac{C}{I} \approx-\frac{\bar{z}_{e}^{2}-\bar{z}_{o}^{2}}{2 \xi \bar{z}_{e} \bar{z}_{o}+j\left(\bar{z}_{e}-\bar{z}_{o}\right)\left(1-\bar{z}_{e} \bar{z}_{o}\right)} .
$$

So, the phase difference at $f=2 f_{1}$ equals

$$
\angle C-\angle I \approx \pi-\tan ^{-1}\left(\frac{\left(\bar{z}_{e}-\bar{z}_{o}\right)\left(1-\bar{z}_{e} \bar{z}_{o}\right)}{2 \xi \bar{z}_{e} \bar{z}_{o}}\right) .
$$




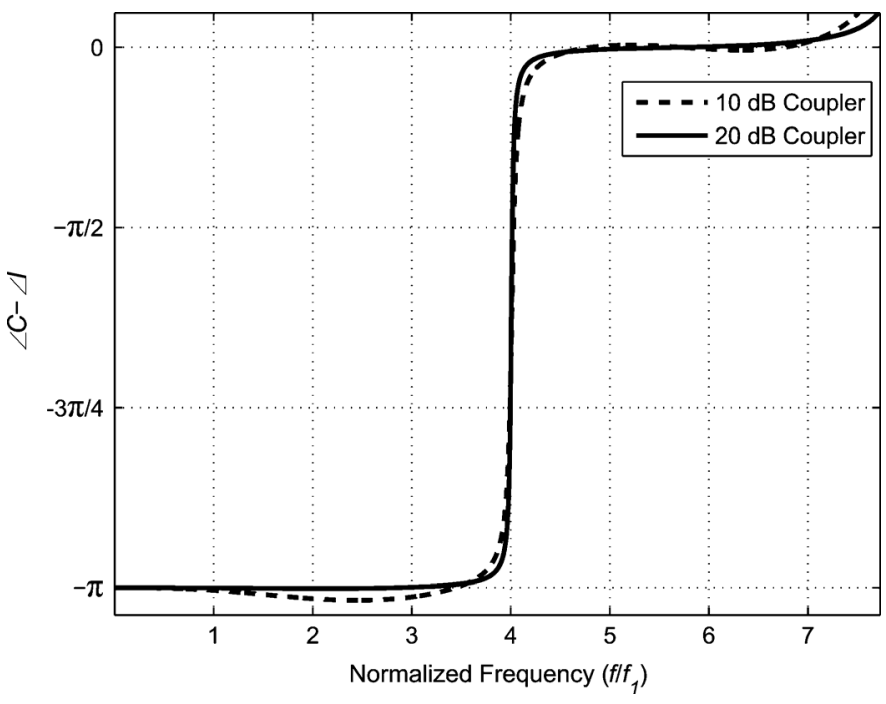

Fig. 2. Phase difference (in radians) between coupling and isolation factors for two couplers specified in Table I.

TABLE I

EXAMPLE COUPLERS.

\begin{tabular}{|l|l|l|}
\hline Coupling & $10 \mathrm{~dB}$ & $20 \mathrm{~dB}$ \\
\hline$b=\beta_{e} / \beta_{O}$ & 1.105 & 1.073 \\
\hline$\overline{z_{e}}$ & 1.365 & 1.10 \\
\hline$\overline{z_{o}}$ & 0.709 & 0.90 \\
\hline Implementation & $W=1.53 \mathrm{~mm}$ & $W=1.79 \mathrm{~mm}$ \\
$e_{r}=3.55, h=0.8 \mathrm{~mm}$ & $S=0.125 \mathrm{~mm}$ & $S=0.99 \mathrm{~mm}$ \\
\hline
\end{tabular}

Using (10) and $\xi=(\pi / 2)(b-1) / b,(21)$ can be rewritten as $b$

$$
\angle C-\angle I \approx \pi-\tan ^{-1}\left(\frac{\left(1-\bar{z}_{o}^{2}\right)\left(\bar{z}_{e}-\bar{z}_{o}\right)}{\pi \bar{z}_{o}\left(\bar{z}_{e}+\bar{z}_{o}\right)}\right) \approx \pi
$$

since $\left(1-\bar{z}_{o}^{2}\right)\left(\bar{z}_{e}-\bar{z}_{o}\right) \ll \pi \bar{z}_{o}\left(\bar{z}_{e}+\bar{z}_{o}\right)$. With low coupling levels, the error term is even smaller. For example, for a $10 \mathrm{~dB}$ coupler with $b=1.1$, (22) gives a phase difference of $\pi-$ $0.022 \pi$, for a $30 \mathrm{~dB}$ coupler with $b=1.1$ the result is $\pi-$ $0.0002 \pi$. Hence the imaginary term in the denominator of (20) can be ignored, and we find the directivity, $D_{2}$, at $f=2 f_{1}$ as

$$
D_{2}=\left|\frac{C}{I}\right| \approx \frac{1}{\pi} \frac{b}{b-1}\left(\frac{\bar{z}_{e}}{\bar{z}_{o}}-\frac{\bar{z}_{o}}{\bar{z}_{e}}\right)
$$

which is smaller than $D_{0}$.

A numerical evaluation of (3) and (4) as depicted in Fig. 2 shows that $(\angle C-\angle I) \approx \pi$ approximation is actually valid along a wide range: $0 \leq f<4 f_{1}$. It is possible to reach a similar conclusion using the network theory (See Appendix). Hence we can write in this frequency range

$$
D=\left|\frac{C}{I}\right|=-\frac{C}{I}
$$

Table I lists the specifications of two couplers that are used in examples. $W$ and $S$ are the width and spacing of the lines. $b=$ $\beta_{e} / \beta_{o}$ ratios are extracted using a microwave circuit simulator ${ }^{2}$ considering a representative dielectric permittivity of $e_{r}=3.55$ and substrate thickness of $0.8 \mathrm{~mm} . \overline{z_{e}}$ and $\overline{z_{o}}$ are calculated using
(8) and the prescribed coupling level. For the $10 \mathrm{~dB}$ coupler, the theoretical phase deviation of $\angle C-\angle I$ from $\pi$ is less than $4.2^{\circ}$ in $0 \leq f<3.8 f_{1}$. For the $20 \mathrm{~dB}$ coupler, the error is smaller than $0.27^{\circ}$ in $0 \leq f<3.2 f_{1}$.

\section{Application of the Phase Relations}

The inherent $\pi$ phase difference between $C$ and $I$ in a wide frequency range can be used to achieve broadband directivity by a cancellation at the isolated port of the couplers. Two methods are explored in the following sections. Short length couplers [14], [18] are utilized as the directivity characteristic is relatively constant for the frequencies lower than the quarter wavelength frequency.

\section{A. Reflected Power Cancellation}

In a conventional coupler, the directivity is highly deteriorated by the unmatched loads at the outputs. This condition is generally faced when one desires to monitor forward and reverse power values using detector circuits at the coupled and isolated ports. If the input reflection of the detectors is not low enough, the directivity is severely reduced. For example, the directivity of a $20 \mathrm{~dB}$ coupler may easily degrade to $12 \mathrm{~dB}$ if the load connected to the coupled port has a return loss of $16 \mathrm{~dB}$. A common solution for this problem is to use two separate couplers, one for the forward, the other for reverse power measurements. Unused ports are terminated perfectly not to degrade the directivity.

On the other hand, if the termination of the unused port is properly chosen, the isolation can be improved. The technique was used in [10] and [11] and narrowband directivity peaks were obtained. In what follows, we present how a broadband directivity improvement can be obtained.

Referring to Fig. 3 and (1), suppose $a_{1}=1, a_{2}=0, a_{4}=0$ and that the coupled port is terminated with a load of reflection coefficient $\Gamma_{x}$ such that $a_{3}=\Gamma_{x} b_{3}$. We can write the modified reflection, $\Gamma_{m}=b_{1} / a_{1}$, as

$$
\Gamma_{m} \approx \Gamma+C^{2} \Gamma_{x} \approx \Gamma
$$

since $|C|^{2}$ is small. The modified isolation, $I_{m}=b_{4} / a_{1}$, is given by

$$
I_{m} \approx I+C \Gamma_{x} e^{-j \beta L} .
$$

Here, the effective propagation constant $\beta$ can be expressed in terms of $\beta_{e}$ using the relations between the effective dielectric permittivity and the even odd mode permittivities given in [19]

$$
\beta=\frac{\beta_{e}+\beta_{o}}{2}=\beta_{e}\left(\frac{b+1}{2 b}\right) .
$$

Equation (26) reveals that the isolation can be improved considerably if $\Gamma_{x}$ can be chosen such that

$$
\Gamma_{x} \approx-\frac{I e^{j \beta L}}{C} \approx \frac{e^{j \beta L}}{D}
$$

where (24) is utilized.

By choosing $a_{1}=0, a_{2}=1, a_{3}=\Gamma_{x} b_{3}$ and $a_{4}=0$, the modified coupling factor, $C_{m}=b_{4} / a_{2}=b_{4}$, is found as

$$
C_{m} \approx C+I \Gamma_{x} e^{-j \beta L}=C\left(1-\frac{I^{2}}{C^{2}}\right) \approx C
$$




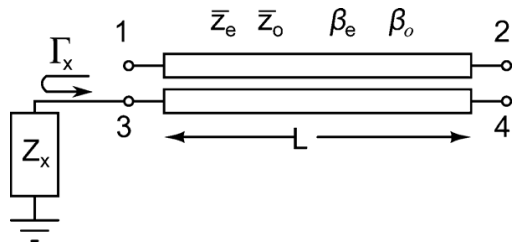

Fig. 3. Coupler with an unmatched load of $Z_{x}$ at port 3 to improve directivity at port 4 using reflected power cancellation scheme.

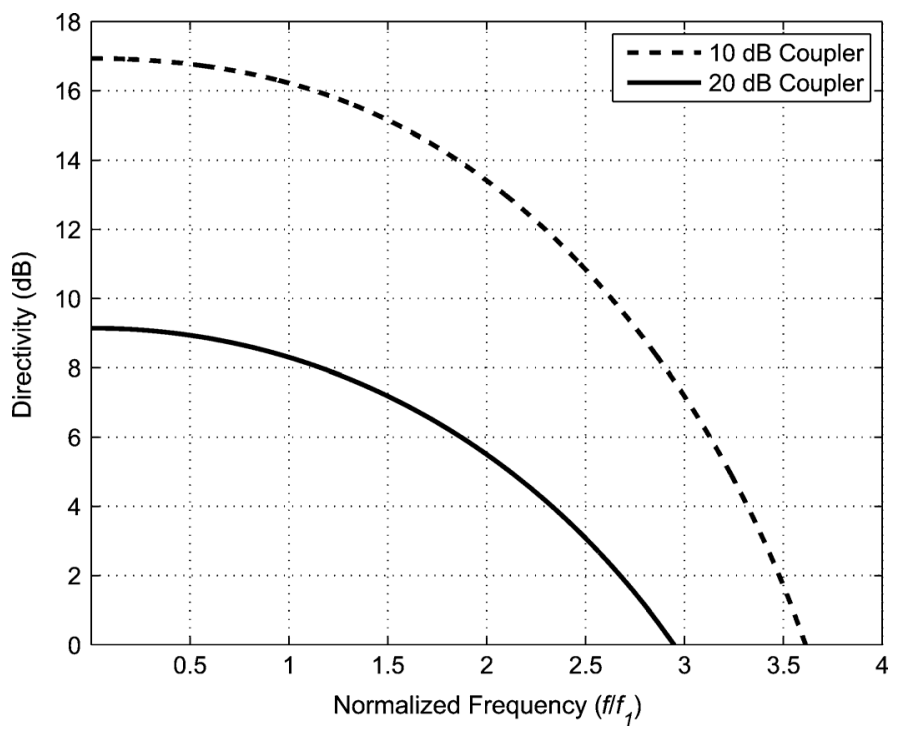

Fig. 4. Directivity characteristic of the two couplers specified in Table I.

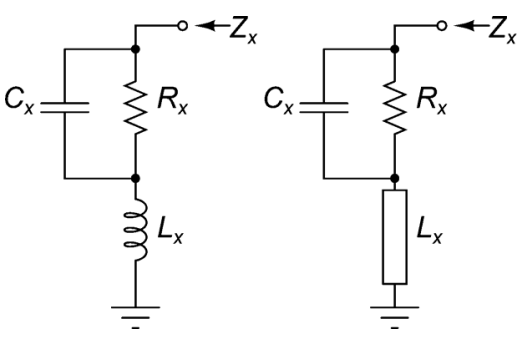

Fig. 5. Lumped element and distributed element versions of proposed circuit for $Z_{x}$. ( $L_{x}$ can be approximated by a shorted transmission line).

since $|I / C|^{2}=1 / D^{2} \ll 1$. We note that in the modified coupler of Fig. 3, the phase difference between $C_{m}$ and $I_{m}$ is no longer $\pi$.

Realization of (28) requires an amplitude response in the form of $1 / D$ in addition to the linear phase term. The couplers with a minimized input return loss have a monotonic decreasing directivity characteristic as shown in Fig. 4. The load impedance shown in Fig. 5 is a good choice to approximately satisfy (28).

The values of the components in Fig. 5 can be found by equating the impedance of the proposed load to the desired load at two frequencies, $f=0$ and $f=f_{a}$. The impedance at $f=0$ is a pure resistance. The directivity, $D_{0}$, at $f=0$ is expressed in (13). So, we can find the required normalized resistor, $\bar{R}_{x}=R_{x} / Z_{0}$ as

$$
\bar{R}_{x}=\frac{1+\frac{1}{D_{0}}}{1-\frac{1}{D_{0}}}=\frac{b \bar{z}_{e}-\bar{z}_{o}}{\frac{1}{\bar{z}_{o}}-\frac{b}{\bar{z}_{e}}} .
$$

Equating the impedances at $f=f_{a}\left(\omega=\omega_{a}\right)$

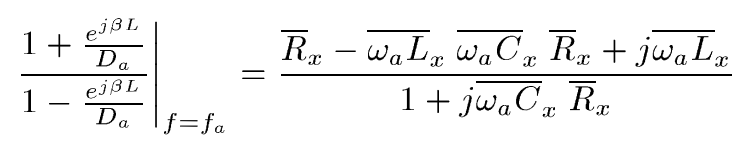

where $D_{a}$ is the directivity at $f_{a}$. At $f=f_{a}, \beta L=\pi f_{a}(b+$ 1) $/ 8 f_{1} b$. The normalized susceptance $\bar{\omega}_{a} C_{x}=Z_{0} \omega_{a} C_{x}$ and reactance $\bar{\omega}_{a} L_{x}=\omega_{a} L_{x} / Z_{0}$ can be calculated by equating the real and imaginary parts of (31) at $f=f_{a}$

$$
\begin{aligned}
& {\overline{\omega_{a} C_{x}}}^{=}=\frac{1}{\bar{R}_{x}} \sqrt{\frac{\bar{R}_{x}\left(D_{a}^{2}-2 D_{a} \cos \theta_{a}+1\right)}{D_{a}^{2}-1}-1} \\
& {\overline{\omega_{a} L_{x}}}=\frac{2 D_{a} \sin \theta_{a}}{D_{a}^{2}-2 D_{a} \cos \theta_{a}+1}+\frac{{\overline{\omega_{a} C}}_{x} \bar{R}_{x}^{2}}{1+{\overline{\omega_{a} C_{x}^{2}}}^{2} \bar{R}_{x}^{2}}
\end{aligned}
$$

with $\theta_{a}=\pi f_{a}(b+1) / 8 f_{1} b$.

$f_{a}$ is varied to get differing amounts of improvement in the directivity. Smaller values of $f_{a}$ give higher directivity but a small bandwidth. Larger values of $f_{a}$ result in a lower directivity with a larger bandwidth. Fig. 6 shows the resulting normalized bandwidth, $f_{B} / f_{1}$, for two different coupling levels as a function of the obtained directivity. Directivity is specified as the minimum value in the frequency range $0<f<f_{B}$. Fig. 7 depicts the required component values to achieve the desired directivity for the same couplers. For example, for the $10 \mathrm{~dB}$ coupler, the directivity is at least $35 \mathrm{~dB}$ in the frequency range $0<f<1.45 f_{1}$ using components $\bar{R}_{x}=1.33, \bar{\omega}_{1} L_{x}=0.6$ and $1 /{\overline{\omega_{1} C}}_{x}=4.4$.

\section{B. Forward Power Cancellation}

Another technique suitable for bandwidth enhancement through the results of the phase difference analysis is the forward power cancellation. An attenuated version of the coupled signal is forwarded to the isolated port to cancel the signal there. The method was used in [12], [13], however the directivity improvement in both works was narrowband. A wideband cancellation is possible as described below.

Fig. 8 shows the proposed structure where an equalizer is implemented using a $\pi$-attenuator with an inductor in one of the arms. The other arm of the attenuator does not have the inductor to keep the input return loss high, since a reflection may reduce the directivity. At high frequencies a properly chosen inductor $L_{1}$ reduces the attenuation to mimic the desired directivity versus frequency characteristics. The extra phase introduced by the equalizer is compensated by unequal length transmission lines, $l_{1}$ and $l_{2}$. Three resistors of value $R_{3}=Z_{0} / 3$ are utilized to combine the signals at port 3 .

Let $T_{1}$ be the frequency dependent complex transmission coefficient of the equalizer and $\Gamma_{1}$ and $\Gamma_{2}$ be the reflection coefficients as shown in Fig. 8. If $\left|\Gamma_{1}\right| \ll\left|T_{1}\right|$, the modified isolation coefficient, $I_{m}^{\prime}=b_{3} / a_{1}$, is given by

$$
I_{m}^{\prime} \approx \frac{\left(I e^{-j \beta l_{2}}+C T_{1} e^{-j \beta l_{1}}\right)}{2}
$$

where $1 / 2$ factor is due to the power combining circuit. So, the required transmission coefficient to nullify $I_{m}^{\prime}$ is

$$
T_{1}=\frac{e^{j \beta\left(l_{1}-l_{2}\right)}}{D} .
$$




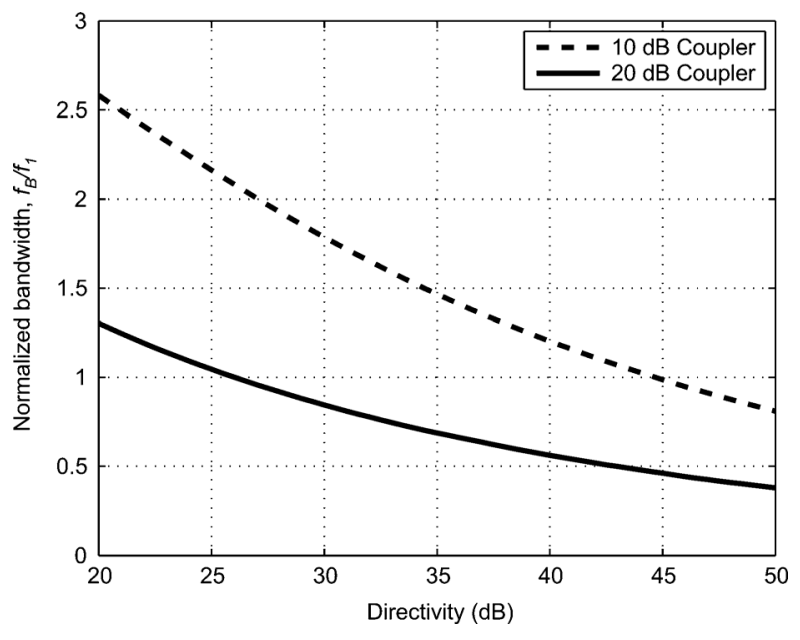

Fig. 6. Normalized bandwidth $\left(f_{B} / f_{1}\right)$ versus directivity with reflected power cancellation technique for the couplers specified in Table I. Directivity is satisfied within the frequency band $0<f<f_{B}$.

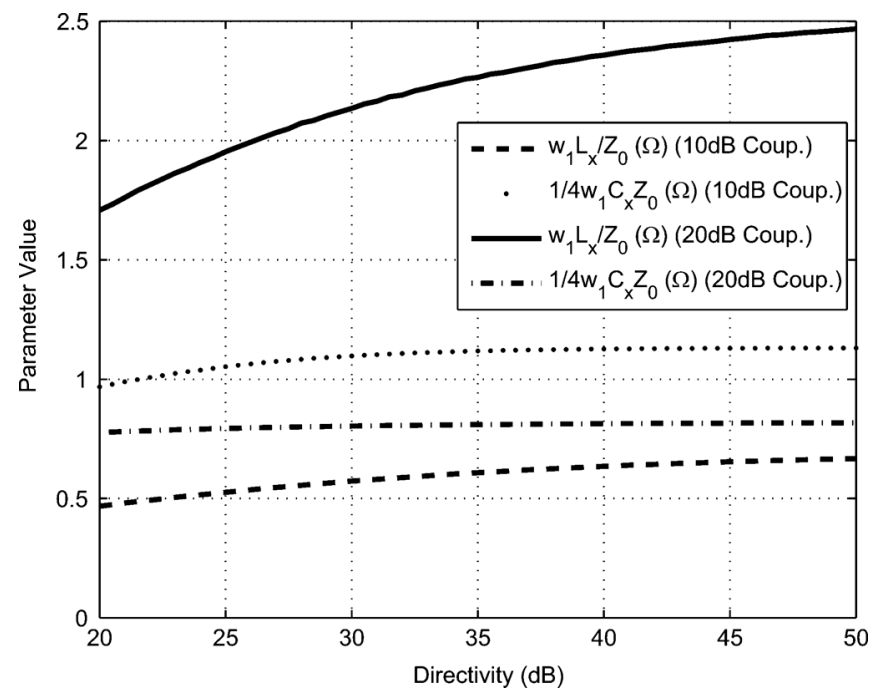

Fig. 7. Normalized reactance values using (32) and (33) to achieve the required directivity in Fig. 6 for two couplers. The normalized reactance values are specified at $f_{1}=\omega_{1} / 2 \pi$. The capacitive reactance is scaled by $1 / 4 . R_{x} / Z_{0}$ is 1.33 for the $10 \mathrm{~dB}$ coupler and 2.07 for the $20 \mathrm{~dB}$ coupler.

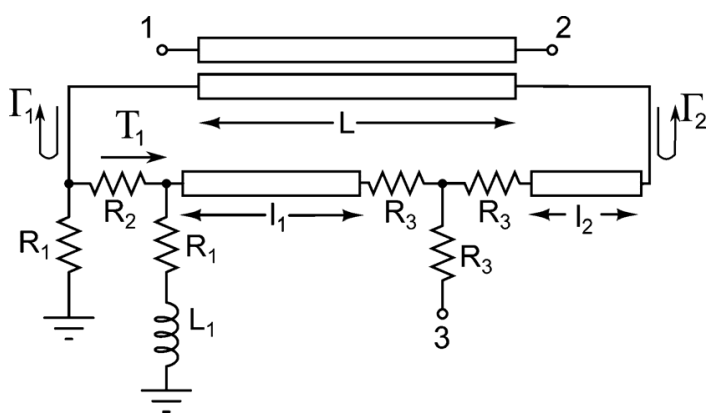

Fig. 8. Coupler with improved directivity at port 3 using forward power cancellation scheme.

Similarly, the modified coupling coefficient, $C_{m}^{\prime}=b_{3} / a_{2}$, can be expressed as

$$
C_{m}^{\prime}=\frac{\left(C e^{-j \beta l_{2}}+I T_{1} e^{-j \beta l_{1}}\right)}{2} \approx \frac{C e^{-j \beta l_{2}}}{2}
$$

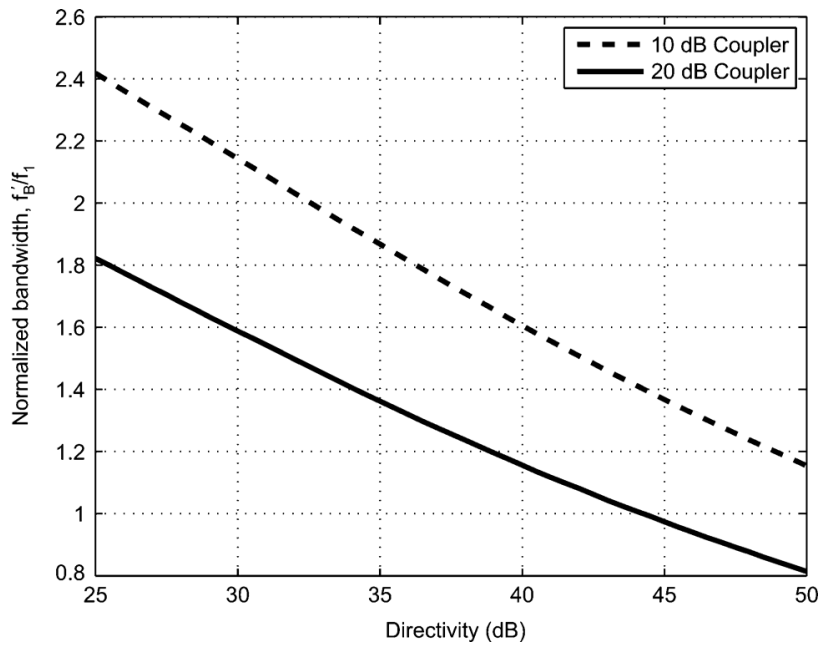

Fig. 9. Normalized bandwidth $\left(f_{B}^{\prime} / f_{1}\right)$ versus directivity with forward power cancellation technique for the couplers specified in Table I. Directivity is satisfied within the frequency band $0<f<f_{B}^{\prime}$.

since we have $\left|T_{1}\right|=1 / D$ from (35) and $\left|I T_{1}\right| \ll|C|$. In other words, the coupling of the forward cancellation scheme is $6 \mathrm{~dB}$ lower than the original coupler. Considering only the first order reflections, the modified input reflection coefficient, $\Gamma_{m}^{\prime}=b_{1} / a_{1}$, can be expressed as

$$
\Gamma_{m}^{\prime} \approx \Gamma+C^{2} \Gamma_{1}+I^{2} \Gamma_{2} \approx \Gamma
$$

where the last approximation is due to small $\left|\Gamma_{1}\right|,\left|\Gamma_{2}\right|, C^{2}$ and $I^{2}$ terms.

Similar to the previous technique, we impose perfect directivity at $f=0$ and $f=f_{a}$. At $f=0$, the equalizer is a pure attenuator. The values of the required resistors can be formulated considering that the attenuation level should be equal to $1 / D_{0}$. The normalized resistor values are

$$
\begin{aligned}
& \overline{R_{1}}=\frac{1+\frac{1}{D_{0}}}{1-\frac{1}{D_{0}}} \\
& \bar{R}_{2}=\frac{D_{0}^{2}-1}{2 D_{0}}
\end{aligned}
$$

where $D_{0}$ is given by (13). The value of $L_{1}$ and the length difference $l_{1}-l_{2}$ can be found by the conditions at $f=f_{a}$. The normalized reactance, ${\overline{\omega_{a}}}_{1}$ is found as

$$
{\overline{\omega_{a}}}_{1}=\sqrt{\frac{\left(\bar{R}_{1}+\bar{R}_{2}+\bar{R}_{1} \bar{R}_{2}\right)^{2}-D_{a}^{2} \bar{R}_{1}{ }^{2}}{D_{a}^{2}-\left(1+\bar{R}_{2}\right)^{2}}}
$$

where $D_{a}$ is the uncompensated directivity at $f=f_{a} . \beta\left(l_{1}-\right.$ $l_{2}$ ) is the phase difference necessary to compensate the positive phase caused by $L_{1}$ specified at $f_{a}$

$\beta\left(l_{1}-l_{2}\right)=\tan ^{-1}\left(\frac{\bar{\omega}_{a} L_{1}}{\bar{R}_{1}}\right)-\tan ^{-1}\left(\frac{\bar{\omega}_{a} L_{1}\left(1+\bar{R}_{2}\right)}{\bar{R}_{1}+\bar{R}_{2}+\bar{R}_{1} \bar{R}_{2}}\right)$.

The couplers listed in Table I are simulated to check the bandwidth capability of the forward power cancellation technique. The results are shown in Fig. 9. A better performance is obtained compared to the reflected power cancellation scheme. For 


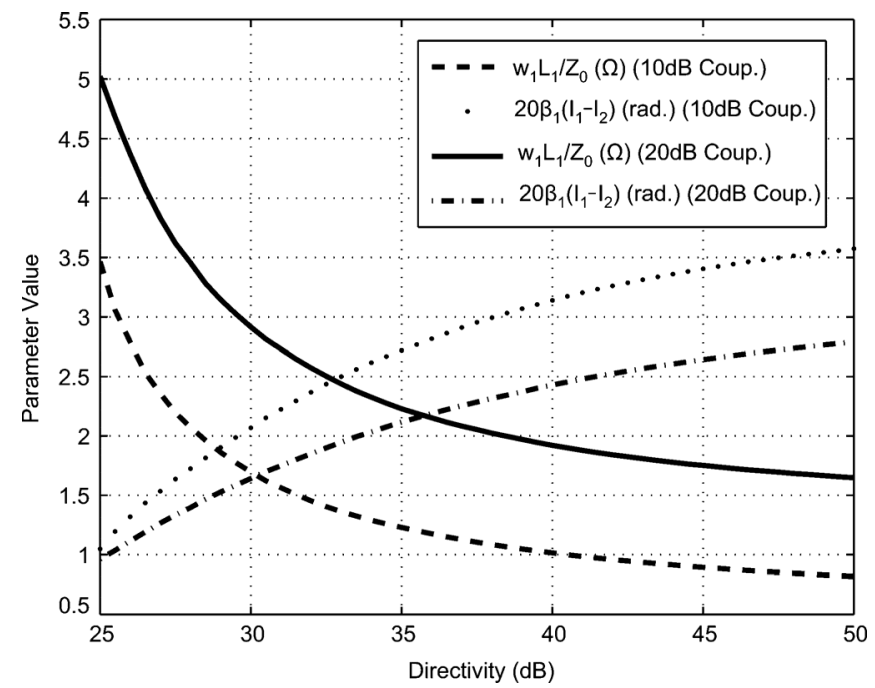

Fig. 10. Normalized values to achieve the directivity for two couplers using the forward power cancellation scheme shown in Fig. 8. The reactance and phase values are specified at $f_{1}$. The phase value is scaled by 20 to improve readability. $\overline{R_{1}}, \overline{R_{2}}$, respectively are $1.33,3.44$ for the $10 \mathrm{~dB}$ coupler and $2.072,1.258$ for the $20 \mathrm{~dB}$ coupler.

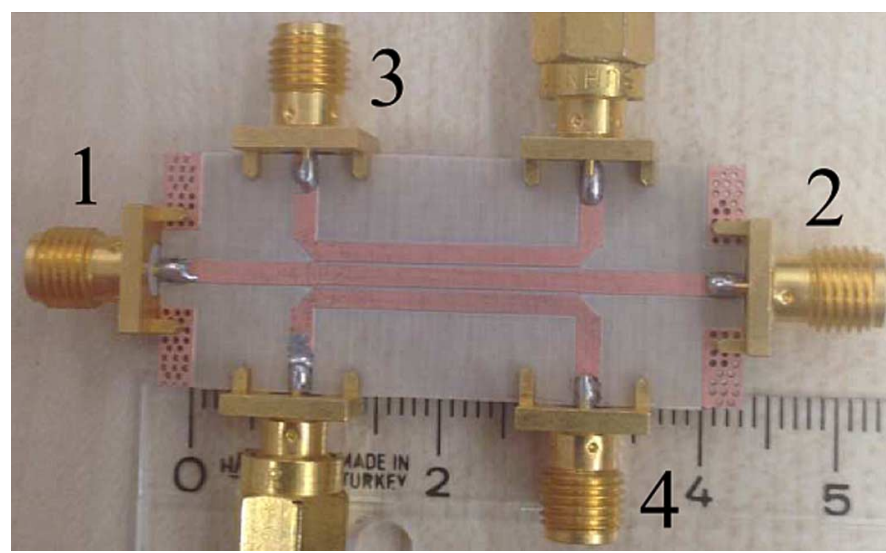

Fig. 11. Photo of the original microstrip coupler.

example, the directivity of the $10 \mathrm{~dB}$ coupler can be increased to $35 \mathrm{~dB}$ in the frequency range $0<f<1.85 f_{1}$. Fig. 10 shows the component values to achieve the required directivity.

\section{EXPERIMENTAL RESULTS}

Couplers are designed and implemented for experimental verification. RO $4003^{3}$ substrate with a thickness of $0.8 \mathrm{~mm}$ is used with a coupled section length of $L=20 \mathrm{~mm}\left(f_{1}=1.12 \mathrm{GHz}\right)$ and a gap of $W=0.3 \mathrm{~mm}$. Coupled lines are added on both sides of the main line ${ }^{4}$ to get independent coupled and isolated ports as depicted in Fig. 11. For input at port 1, the coupled port is \#3 and the isolated port is \#4. For this coupler $z_{e}=62 \Omega$, $z_{o}=39.4 \Omega, b=1.098, D_{0}=14 \mathrm{~dB}$ and $|C|=-13 \mathrm{~dB}$ at $f=2 f_{1}$. Fig. 12 shows the calculated (from (3) and (4)) and measured phase differences between the coupling $\left(S_{31}\right)$ and the isolation $\left(S_{32}\right)$. The variation in the measured phase difference

\footnotetext{
${ }^{3}$ Rogers Corp. Rogers, CT 06263, USA, http://www.rogerscorp.com

${ }^{4}$ Since the coupling is weak, analytical results of previous sections are also valid for this three-line coupler.
}

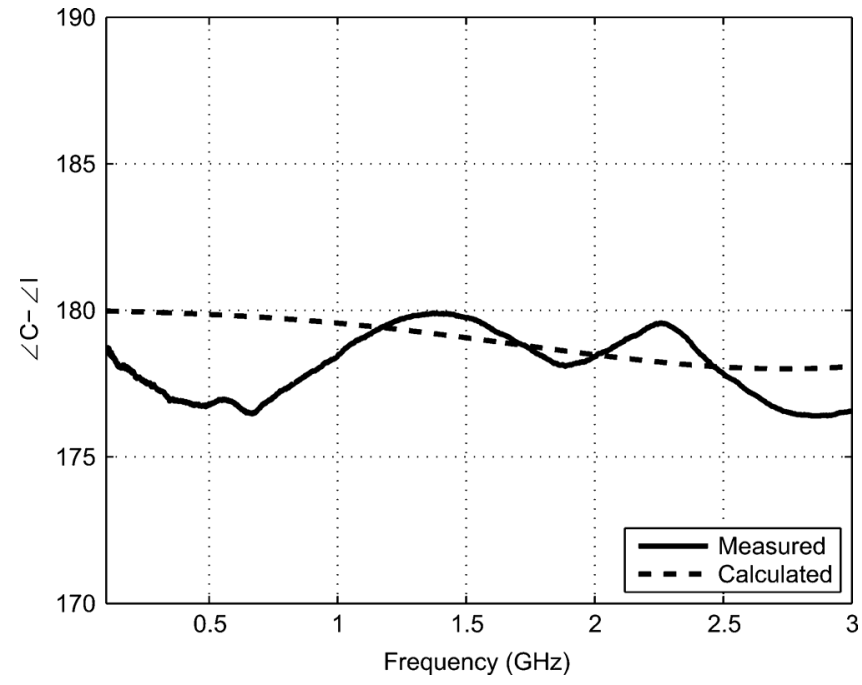

Fig. 12. Calculated and measured phase differences (in degrees) between the coupling and the isolation for the original coupler.

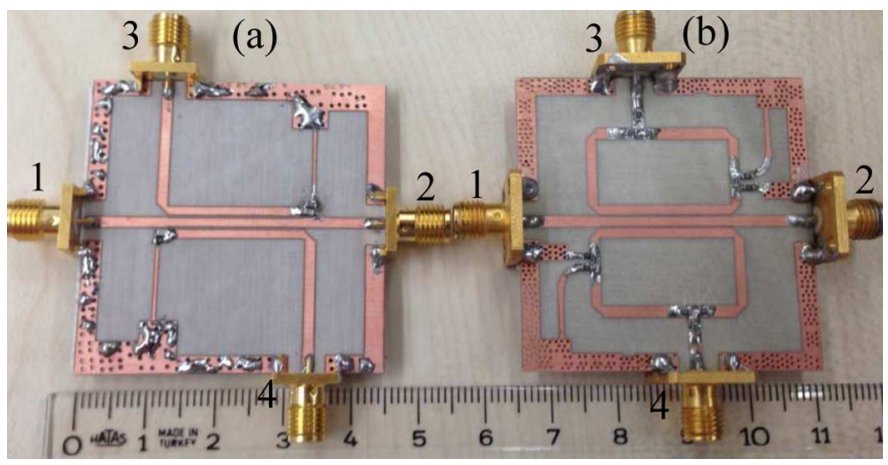

Fig. 13. Photo of the couplers with reflected power cancellation and forward power cancellation schemes.

is higher than expected, possibly because of the small reflections at $90^{\circ}$ bends and at the termination resistor in the isolation measurement.

The reflected power and the forward power cancellation schemes are implemented as shown in Fig. 13. The component values are found from the equations of Section-III. With less ambitious directivity goals, these values can be used directly. But for higher directivity values, a modification in values is necessary to take care of the parasitic effects such as transmission line bends, junction discontinuities, via hole inductors, unintended couplings and the parasitics of the lumped elements. An optimization is performed using the microwave circuit simulator and an electromagnetic simulator ${ }^{5}$ for the final values.

For the reflected power cancellation scheme, the coupler is designed to give a directivity of $40 \mathrm{~dB}$. For this directivity target, we estimate from Fig. 6 a bandwidth of $f_{B}=0.9 f_{1}=1 \mathrm{GHz}$. Component values of the termination can be calculated from (30), (32) and (33) as $R_{x}=74.8 \Omega, C_{x}=0.73 \mathrm{pF}$ and $L_{x}=$ $7.3 \mathrm{nH}$. The component values of $R_{x}=75 \Omega$ and $C_{x}=0.70 \mathrm{pF}$ are used at the experimental step. $L_{x}$ is realized with a microstrip line of $0.6 \mathrm{~mm}$ width and $10 \mathrm{~mm}$ length.

${ }^{5}$ SONNET Software, North Syracuse NY 13212, USA, http://www.sonnetsoftware.com 


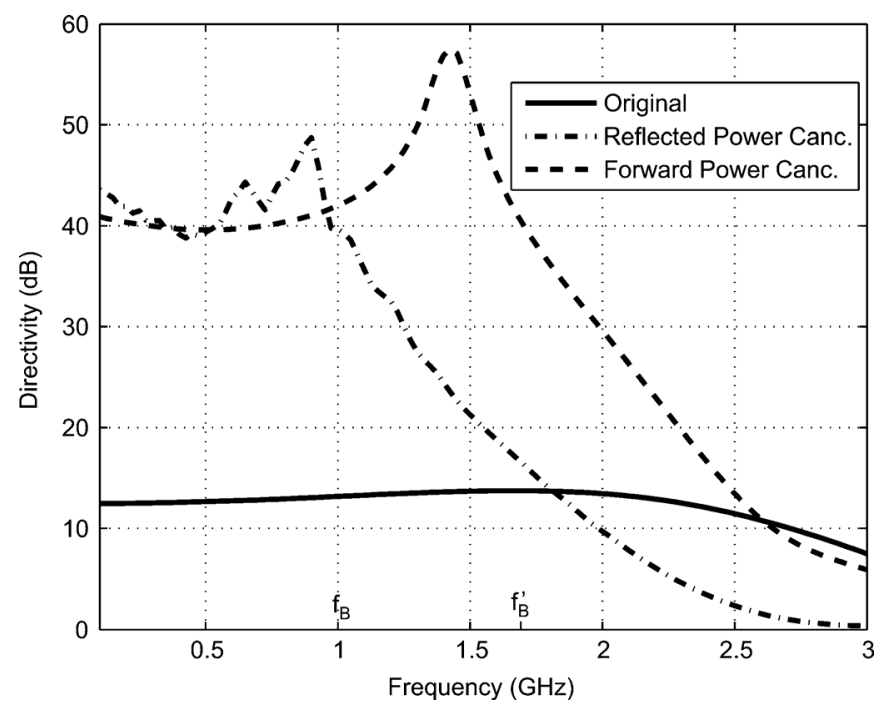

Fig. 14. Directivity of the original, reflected power cancellation and forward power cancellation schemes as found by the microwave circuit simulator.

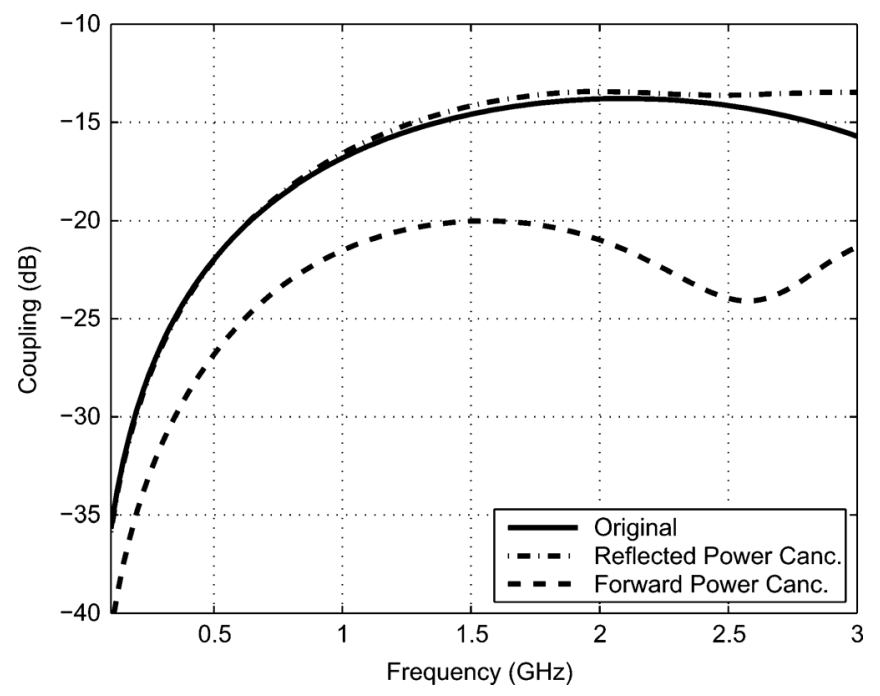

Fig. 15. Coupling of the original, reflected power cancellation and forward power cancellation schemes as found by the microwave circuit simulator.

For the forward power cancellation technique, Fig. 9 predicts a bandwidth of $f_{B}^{\prime}=1.5 f_{1}=1.7 \mathrm{GHz}$ for a directivity of $40 \mathrm{~dB}$. From (38), (39), (40), (41), the required component values are $R_{1}=74.8 \Omega, R_{2}=121 \Omega, L_{1}=8.5 \mathrm{nH}$ and $\beta_{1}\left(l_{1}-l_{2}\right)=0.15$ implying $l_{1}-l_{2}=3.8 \mathrm{~mm}$. Following the optimization, the component values are modified. In the experimental step, $75 \Omega-178 \Omega-82.5 \Omega$ resistor combination results in a better directivity response. $L_{1}$ is realized with a shorted microstrip line of $0.8 \mathrm{~mm}$ width and $13 \mathrm{~mm}$ length.

Simulated directivity responses are displayed in Fig. 14 verifying the predicted $f_{B}$ and $f_{B}^{\prime}$ values for both schemes. Fig. 15 presents the coupling responses. We note that the coupling value itself is not constant for $f$ less than $f_{B}$ or $f_{B}^{\prime}$, a characteristic of short length couplers [18]. In this frequency range, the coupling of the reflected cancellation scheme follows that of the original coupler, while the coupling of the forward power cancellation case is approximately $6 \mathrm{~dB}$ lower as expected. The simulated input return losses of the reflected and the forward cancellation

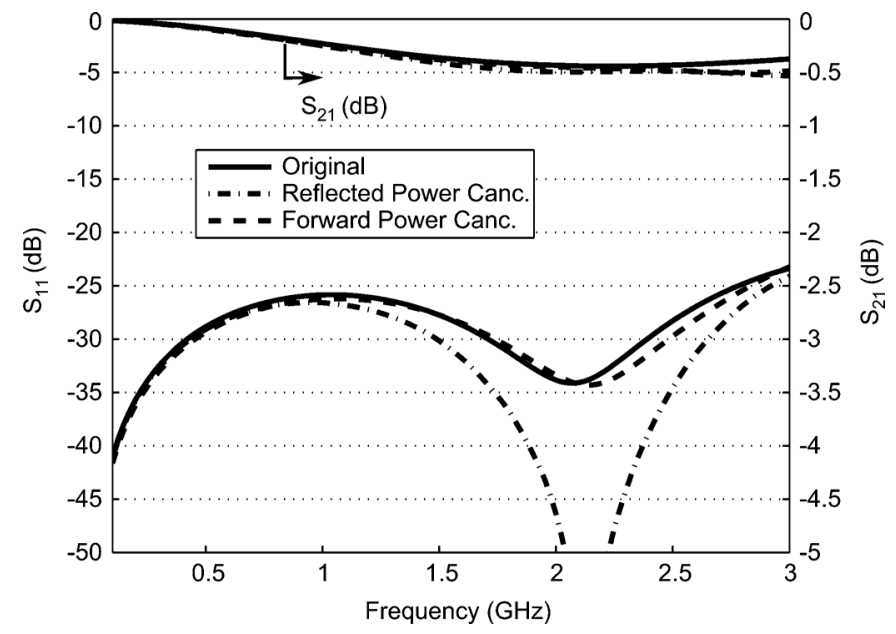

Fig. 16. Return loss and insertion loss characteristics of the original, reflected power cancellation and forward power cancellation schemes as found by the microwave circuit simulator.

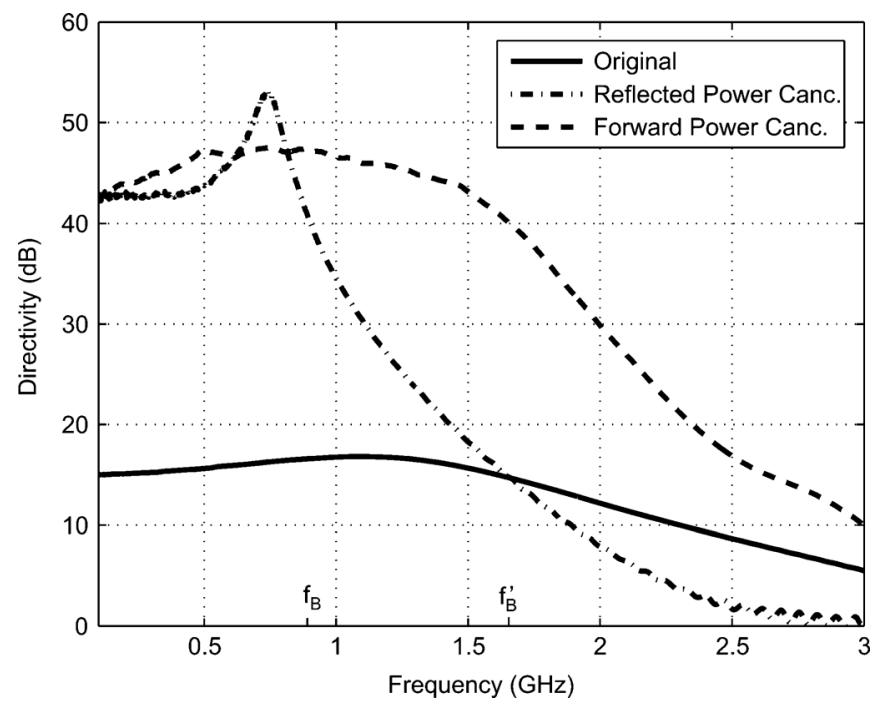

Fig. 17. Measured directivity of the original, reflected power cancellation and forward power cancellation schemes.

schemes are close to the original one as seen in Fig. 16. The insertion losses are better than $0.5 \mathrm{~dB}$ for both cases.

Measured directivity responses are given in Fig. 17. The reflected power cancellation scheme has directivity of $40 \mathrm{~dB}$ in the band $0<f<0.9 \mathrm{GHz}$. On the other hand, the forward power cancellation method gives the same directivity in the band $0<f<1.7 \mathrm{GHz}$. Figs. 18 and 19 show the measured coupling, return loss and insertion loss responses. The measurements are in good agreement with the simulation results.

\section{CONCLusions}

An interesting phase relation of microstrip coupled line couplers is explored. It is shown that the phase difference between the coupling and the isolation coefficients is very close to $\pi$ in a wide band. This characteristic is suitable for directivity enhancement using two different methods proposed in this paper. The required component values for both methods can be calculated by the given analytical expressions.

For the given directivity, the reflected power cancellation scheme gives a smaller bandwidth compared to the forward 


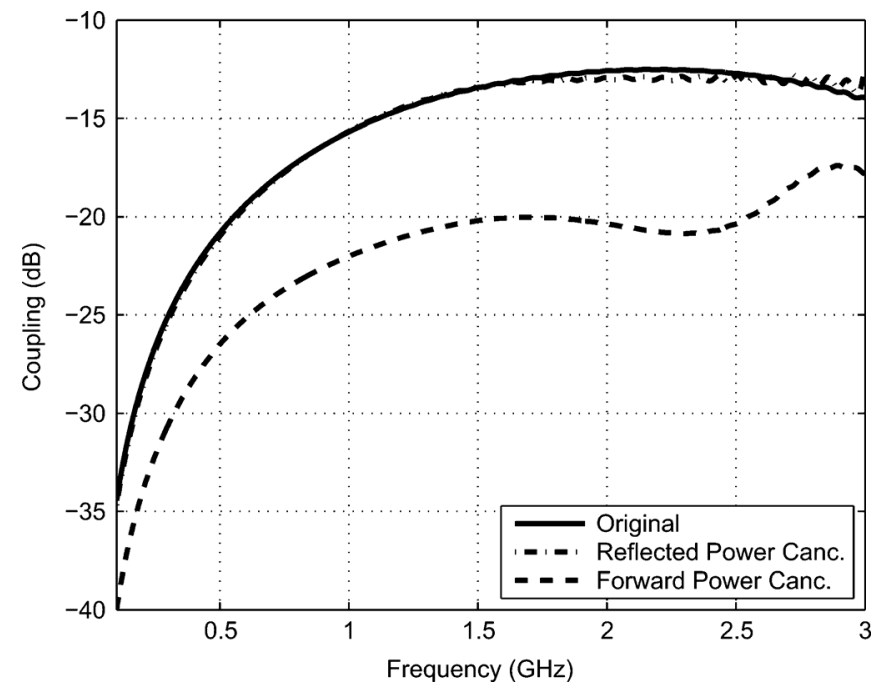

Fig. 18. Measured coupling of the original, reflected power cancellation and forward power cancellation schemes.

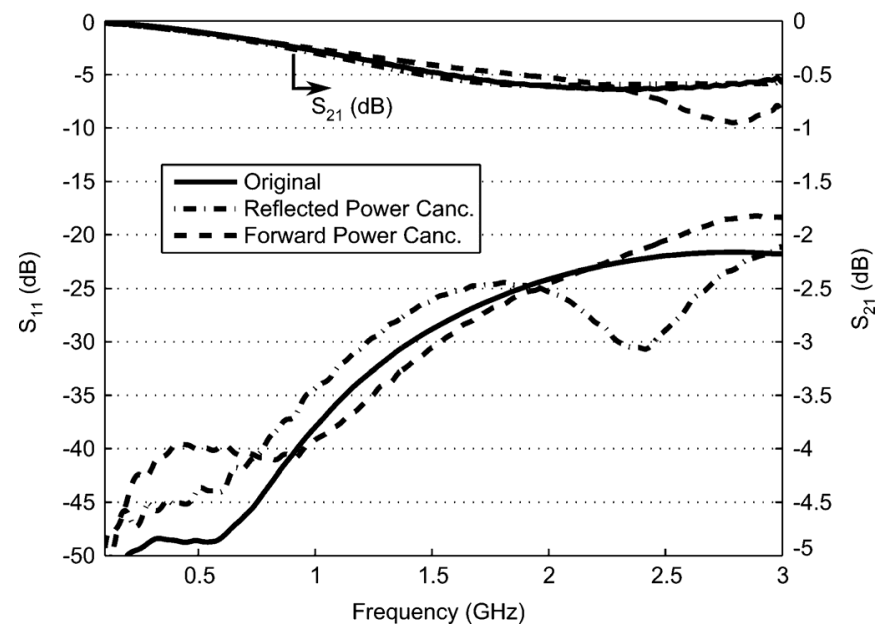

Fig. 19. Measured return loss and insertion loss characteristic of the original, reflected power cancellation and forward power cancellation schemes.

power cancellation scheme. But its implementation is easier and requires less optimization.

\section{APPENDIX \\ Phase DifFERENCE BETWEen COUPLING AND ISOLATION COEFFICIENTS OF A DiRECTIONAL COUPLER}

Network analysis yields that a reciprocal, lossless, matched four-port network is a directional coupler with infinite directivity [15]. Unfortunately, this is an idealized situation: For real life couplers the directivity is finite since the four-port matching constraint is not satisfied perfectly although the reciprocity condition is valid and the loss can be kept at very small values.

The lossless condition dictates the following relation between the S-parameters [15]

$$
\sum_{k=1}^{4} S_{k m} S_{k n}^{*}= \begin{cases}1, & \text { if } m=n \\ 0, & \text { otherwise }\end{cases}
$$

where * denotes the complex conjugate operation. Using (1) and (42), we find

$$
\begin{aligned}
|\Gamma|^{2}+|T|^{2}+|I|^{2}+|C|^{2} & =1 \\
\Gamma T^{*}+T \Gamma^{*}+C I^{*}+I C^{*} & =2\left(\mathfrak{R e}\left\{\Gamma T^{*}\right\}+\mathfrak{R e}\left\{C I^{*}\right\}\right)=0 \\
\Gamma C^{*}+C \Gamma^{*}+T I^{*}+I T^{*} & =2\left(\mathfrak{R e}\left\{\Gamma C^{*}\right\}+\mathfrak{R e}\left\{T I^{*}\right\}\right)=0 \\
\Gamma I^{*}+I \Gamma^{*}+T C^{*}+C T^{*} & =2\left(\mathfrak{R e}\left\{\Gamma I^{*}\right\}+\mathfrak{R e}\left\{T C^{*}\right\}\right)=0
\end{aligned}
$$

where $\mathfrak{R e}\{\}$ indicates the real part operation.

Let us consider a weakly coupled coupler with a coupling coefficient

$$
|C| \ll 1
$$

Obviously, the isolation coefficient is even smaller

$$
|I|<|C| \ll 1
$$

If the coupler is designed to have a low reflection coefficient

$$
|\Gamma| \ll 1 .
$$

From (43), (47), (48) and (49) we find

$$
|T| \approx 1 .
$$

Using (48), (49) we find $\mathfrak{R e}\left\{\Gamma I^{*}\right\} \approx 0$ is negligible. From (46) we must also have $\mathfrak{R e}\left\{T C^{*}\right\} \approx 0$. Therefore,

$$
\angle T-\angle C=\frac{ \pm \pi}{2}
$$

which is a well known characteristic of the couplers. Using (50) and (51) we rewrite (44) and (45) as

$$
\begin{aligned}
\mp|\Gamma| \sin (\angle \Gamma-\angle C)+|I||C| \cos (\angle I-\angle C) & =0 \\
|\Gamma||C| \cos (\angle \Gamma-\angle C) \mp|I| \sin (\angle I-\angle C) & =0 .
\end{aligned}
$$

Rearranging these equations we get

$$
\left(\frac{|I| \sin (\angle I-\angle C)}{|\Gamma||C|}\right)^{2}+\left(\frac{|I||C| \cos (\angle I-\angle C)}{|\Gamma|}\right)^{2}=1 .
$$

Since $\cos ^{2}(\angle I-\angle C)+\sin ^{2}(\angle I-\angle C)=1$ we arrive at

$$
\angle I-\angle C=\tan ^{-1} \sqrt{\frac{1-\left|\frac{I C}{\Gamma}\right|^{2}}{\left|\frac{I}{\Gamma C}\right|^{2}-1}} \pm n \pi .
$$

For example, in a microstrip coupler with a directivity of 14 $\mathrm{dB}$, coupling of $20 \mathrm{~dB}$ and return loss of $30 \mathrm{~dB}$, the deviation of $\angle I-\angle C$ from $n \pi$ is less than 0.16 radians $\left(9.4^{\circ}\right)$. If the return loss is $40 \mathrm{~dB}$, the deviation reduces to 0.05 radians $\left(2.9^{\circ}\right)$. 


\section{REFERENCES}

[1] I. B. R. Mongia and P. Bhartia, RF and Microwave Coupled-Line Circuits. Boston, MA, USA: Artech House, 1999.

[2] A. Podell, "A high directivity microstrip coupler technique," in Proc. Microwave Symp., G-MTT 1970 Int., May 1970, pp. 33-36.

[3] B. Sheleg and B. Spielman, "Broad-band directional couplers using microstrip with dielectric overlays," IEEE Trans. Microw. Theory Tech., vol. 22, no. 12, pp. 1216-1220, Dec. 1974.

[4] J. Klein and K. Chang, "Optimum dielectric overlay thickness for equal even- and odd-mode phase velocities in coupled microstrip circuits," Electron. Lett., vol. 26, no. 5, pp. 274-276, Mar. 1990.

[5] M. Dydyk, "Microstrip directional couplers with ideal performance via single-element compensation," IEEE Trans. Microw. Theory Tech., vol. 47, no. 6, pp. 956-964, Jun. 1999.

[6] S. March, "Phase velocity compensation in parallel-coupled microstrip," in Proc. Microwave Symp. Digest,1982 IEEE MTT-S Int., Jun. 1982, pp. 410-412.

[7] S. Lee and Y. Lee, "A design method for microstrip directional couplers loaded with shunt inductors for directivity enhancement," IEEE Trans. Microw. Theory Tech., vol. 58, no. 4, pp. 994-1002, Apr. 2010.

[8] J. Muller, M. Pham, and A. Jacob, "Directional coupler compensation with optimally positioned capacitances," IEEE Trans. Microw. Theory Tech., vol. 59, no. 11, pp. 2824-2832, Nov. 2011.

[9] Y. Wu, W. Sun, S.-W. Leung, Y. Diao, K.-H. Chan, and Y.-M. Siu, "Single-layer microstrip high-directivity coupled-line coupler with tight coupling," IEEE Trans. Microw. Theory Tech., vol. 61, no. 2, pp. 746-753, Feb. 2013.

[10] J. Muller and A. Jacob, "Complex compensation of coupled line structures in inhomogeneous media," in Proc. Microw. Symp. Dig., Jun. 2008, pp. 1007-1010.

[11] W.-K. Kim, M.-Q. Lee, J.-H. Kim, H. s. Lim, J.-W. Yu, B.-J. Jang, and J.-S. Park, "A passive circulator with high isolation using a directional coupler for RFID," in Proc. Microw. Symp. Dig., Jun. 2006, pp. $1177-1180$.

[12] S. Bensmida and F. Ghannouchi, "New high directivity coupler design using feed-forward compensation technique," in Proc. 38th Eur. Microwave Conf., Oct. 2008, pp. 191-194.

[13] K. Yamauchi, A. Inoue, and M. Miyazaki, "High directivity quarterwave microstrip coupler with cancellation circuit of wilkinson divider," in Proc. Microw. Conf., Oct. 2008, pp. 195-198.

[14] K. Yamamoto, H. Kurusu, S. Suzuki, and M. Miyashita, "High-directivity enhancement with passive and active bypass circuit techniques for gaas mmic microstrip directional couplers," IEEE Trans. Microw. Theory Tech., vol. 59, no. 12, pp. 3095-3107, Dec. 2011.

[15] D. M. Pozar, Microwave Engineering. New York, NY, USA: Wiley, 1998.

[16] R. E. Collin, Foundations for Microwave Engineering. New York, NY, USA: McGraw-Hill Inc, 1992.
[17] M. Kirschning and R. Jansen, "Accurate wide-range design equations for the frequency-dependent characteristic of parallel coupled microstrip lines," IEEE Trans. Microw. Theory Tech., vol. 32, no. 1, pp. 83-90, 1984.

[18] Y. Zhu, J. Zhang, H. Zhu, J. Cheng, and J. Li, "Compact microstrip line directional coupler with high directivity," J. Electromag. Waves Applicat., vol. 26, no. 11-12, pp. 1619-1623, 2012.

[19] A. Eroglu and J. K. Lee, "The complete design of microstrip directional couplers using the synthesis technique," IEEE Trans. Instrum. Meas., vol. 57 , no. 12 , pp. 2756-2761, Dec. 2008.

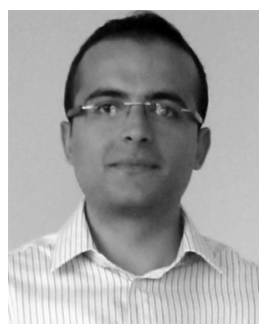

Vahdettin Tas received the B.S. degree from Middle East Technical University, Ankara, Turkey, in 2007 and the M.S. degree from Bilkent University, Ankara, Turkey, in 2009, both in electrical engineering, where he is currently pursuing the Ph.D. degree.

In 2009, he was with the Elecgtrical Engineering and Computer Science Department of UC Berkeley as a Research Assistant. From 2010 to 2011, he worked as an RF design engineer at Meteksan Defense Inc. Since 2011 he is with the Power Amplifier Technologies Divison, Aselsan Inc. His current research interests include broadband and efficient power amplification, power coupling and power combining techniques.

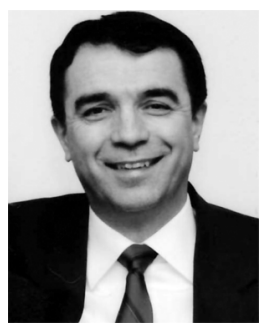

Abdullah Atalar (F'10) received B.S. degree from Middle East Technical University, Ankara, Turkey, in 1974, and the M.S. and Ph.D. degrees from Stanford University, Stanford, CA, USA, in 1976 and 1978, respectively, all in electrical engineering. He worked in Hewlett Packard Labs, Palo Alto, CA, USA, in 1979.

From 1980 to 1986 , he was on the faculty of the Middle East Technical University as an Assistant Professor. In 1986, he joined the Bilkent University as the chairman of the Electrical and Electronics Engineering Department and served in the founding of the Department where he is currently a Professor. In 1995 he was a Visiting Professor of Stanford University. From 1996 to 2010, he was the Provost of Bilkent University. He is presently the Rector of the same university. His current research interests include micromachined devices and microwave electronics.

Dr. Atalar was awarded the Science Award of TUBITAK in 1994. 